\title{
Parasitoid-Induced Cellular Immune Deficiency in Drosophila ${ }^{a}$
}

\author{
TAHIR M. RIZKI AND ROSE M. RIZKI \\ Department of Biology \\ The University of Michigan \\ Ann Arbor, Michigan 48109-1048
}

\section{INTRODUCTION}

The immune response relies on the ability to distinguish non-self from self. Because endoparasites are foreign entities in their hosts, they must circumvent the hosts' capacity to recognize non-self in order to survive in the alien environment. How endoparasites evade detection and destruction by the host's immune system can reveal factors that are important to a successful defense response by the host. The variety of mechanisms used by endoparasites to bypass the challenges imposed by the insect host's cellular defense can be classified in two categories: those that avoid exciting the defense response and those that combat elements of the defense system.

Some parasites evade host hemocytes by residing within host tissues. For example, parasitic wasps (parasitoids) and parasitic nematodes develop beneath the gut epithelium or within ganglia of the central nervous system. ${ }^{1-3}$ Other parasites avoid harmful effects of host hemocytes by utilizing developmental stages of the host in which immune responses are less likely to occur. ${ }^{2,3}$ Parasites that develop within the host hemocoel may have surface coatings that repel, or do not arouse, a hemocyte response. That the thick fibrous layer on braconid Cardiochiles nigriceps eggs thwarts hemocyte attack was demonstrated by experimental removal of this surface layer to render the eggs susceptible to encapsulation by host hemocytes. ${ }^{4}$ Likewise, surface materials on Venturia canescens eggs are responsible for preventing encapsulation. ${ }^{5,6}$ In this parasitoid, $130-$ to $140-\mathrm{nm}$ particles secreted by the calyx cells of the reproductive system adhere to long projections from the egg chorion. ${ }^{7}$ The particles persist on the eggs up to 60 hours after oviposition in the host and are present on first instar larvae as well. The viruslike particles contain glycoproteins but no detectable nucleic acid. ${ }^{8}$ Antibodies raised against purified Venturia particle proteins cross-react to a $42-\mathrm{kDa}$ protein localized in the host fat body. Most of the 42-kDa protein is synthesized in host hemocytes and secreted into the hemolymph where it adheres to the surface layer of the fat body. ${ }^{9}$ The authors concluded that the viruslike particles on the parasitoid eggs confer passive resistance to host hemocytes by virtue of the antigenic determinants they share with the host. This conclusion is compatible with the fact that foreign objects other than the parasitoid eggs are encapsulated in parasitized hosts. ${ }^{10}$ In other parasitoid-host interactions, venom acts

\footnotetext{
${ }^{a}$ This research was supported by grants from the National Institutes of Health and the
} National Science Foundation. 
synergistically with fibrous egg coats to inhibit encapsulation by host hemocytes without disrupting encapsulation of other foreign particles. ${ }^{11,12}$

Although encapsulation by hemocytes contains and destroys foreign objects, this defense reaction need not always be fatal for parasitoid eggs. The eggs of Leptopilina boulardi (strain L104), a habitual parasitoid of Drosophila melanogaster, adhere to the surfaces of host tissues, generally the larval gut. ${ }^{13}$ Host hemocytes attach to the egg surfaces exposed to the hemocoel but cannot penetrate around the region of the egg attached tightly to the underlying host tissue to completely surround the eggs. The incomplete capsules are not effective in preventing escape of the parasitoid larvae, which move actively at hatching and rupture the partial capsules. This means of defense against host hemocytes is not highly effective, because survival of L104 parasitoids in Drosophila hosts is poor compared with parasitoids employing other defense strategies. ${ }^{14.15}$

In contrast to passive mechanisms for protection against host hemocytes, some parasitoids interfere with the cellular defense system of the host to ensure a suitable environment for the development of their eggs. In these insects, accessory organs of the female reproductive system synthesize materials that are coinjected with eggs into the host. The substances prevent encapsulation of not only the parasitoid eggs but also, in some cases, other foreign objects in the host hemocoel as well. That such accessory gland secretions affect hemocytes is suggested by visible effects on cell morphology in parasitized hosts. ${ }^{16}$ More than a single factor may be required to affect host hemocytes; for example, both calyx and venom fluid of Microplitis mediator are necessary to inhibit filopodial elongation of the host hemocytes. ${ }^{17}$

Polydnaviruses that replicate in calyx cells of ichneumonid and braconid parasitoids and are injected into hosts at oviposition inhibit encapsulation of wasp eggs and play several other roles in modifying host physiology for the benefit of the developing parasitoids. ${ }^{18.19}$ The viruses enter a variety of host tissues, including muscle, fat body, tracheal epithelium, and hemocytes. ${ }^{20}$ Expression of polydnaviruses is detectable in hosts shortly after parasitization, but the viruses do not replicate in the parasitized hosts. ${ }^{21-23}$ How the polydnaviruses interfere with encapsulation is not known. Impairment of hemocyte function in parasitized hosts is suggested by studies demonstrating suppression of the in vitro spreading behavior of hemocytes by calyx fluid and polydnaviruses. ${ }^{24-26}$

The most striking instance of hemocyte modification has been reported for Drosophila melanogaster larvae parasitized by Leptopilina heterotoma. ${ }^{27}$ This parasitoid uses viruslike particles (VLPs) produced in an accessary gland (long gland), not in the calyx cells, to disrupt specifically the capsule-forming hemocytes of the host. ${ }^{28,29}$ An evolutionary relationship between polydnaviruses in the calyx and components in venom glands of the reproductive system has been suggested. ${ }^{30.31}$ Therefore, analysis of the $D$. melanogaster-L. heterotoma interaction is interesting and desirable for seeking relationships among the factors used by parasitoids to subjugate host cellular defenses. In addition, this parasitoid-host system offers a unique opportunity to study effects of parasitization on host hemocytes because the type of hemocytes and their responses to foreign materials in the hemocoel of the $D$. melanogaster larva have been studied extensively using mutant strains (see reviews in Rizki and Rizki ${ }^{32,33}$ ). By using these mutant strains as hosts for parasitoids, the effects of parasitization on host hemocytes can be surmised. 


\section{HEMOCYTES OF DROSOPHILA LARVAE}

Two classes of hemocytes are found in normal Drosophila larvae, the plasmatocytes, which are phagocytic cells, and the crystal cells, which have prominent cytoplasmic paracrystalline inclusions containing phenol oxidases. ${ }^{32,33}$ At pupariation, plasmatocytes undergo morphological transformation (or differentiation) from the typical spherical shape to an extremely flattened, disklike cell called the lamellocyte. ${ }^{34,35}$ The leaflike lamellocyte is ideally suited for its function, which is to wrap around large foreign objects to form cellular capsules.

In Drosophila larvae with melanotic tumor genes, lamellocyte differentiation is induced by metabolic products from aberrant tissues and the larvae have many lamellocytes circulating in the hemolymph. ${ }^{36}$ After the surfaces of the abnormal tissues show changes, lamellocytes begin to layer around them in a manner similar to the encapsulation of large foreign objects. ${ }^{37,38}$ Multilayered, cellular capsules in Drosophila larvae are hardened and darkened due to the phenol oxidases of the crystal cells. ${ }^{39-41}$

Evidence for the roles of the crystal cells in the hardening and blackening processes has been obtained by genetically blocking the melanosis of tissues in melanotic tumor mutants. In double-mutant combinations using genes that either cause a deficiency or an absence of phenol oxidases, such as black cell or lozenge mutant alleles, encapsulation of the aberrant tissues created by the melanotic tumor gene proceeds. Nevertheless, the darkening and hardening processes dependent on crystal cell function are interrupted owing to the presence of the mutant genes blocking the availability of phenol oxidases.

\section{HEMOCYTE CHANGES INDUCED BY PARASITIZATION}

L. heterotoma females oviposit in the hemocoel of Drosophila larvae. The freefloating eggs are about $50 \times 600 \mu \mathrm{m}$, too large to be engulfed by plasmatocytes so they should be susceptible to encapsulation by lamellocytes. When Walker ${ }^{42}$ allowed L. heterotoma females to oviposit in melanotic tumor larvae, she found that the encapsulation of tissues by lamellocytes to form melanotic tumors was suppressed and that the parasitoid eggs remained free of encapsulating lamellocytes as well. This generalized blockage of encapsulation in parasitized Drosophila hosts was later confirmed, ${ }^{13,43}$ and the cause of the inhibition of lamellocyte activity was identified. ${ }^{27,28}$

Two to four hours after melanotic tumor larvae are parasitized by L. heterotoma, the lamellocytes in the hemocoel lose their discoidal shape and become bipolar cells. ${ }^{27}$ The change in cell morphology is correlated with changes in cell adhesion. Disk-shaped lamellocytes are sticky cells that adhere and clump together. The bipolar lamellocytes are nonadherent, so it is clear that they are not able to attach to foreign objects or other lamellocytes to form cellular capsules. The affected lamellocytes in parasitized Drosophila larvae undergo degenerative changes involving loss of cytoplasm at the elongating tips. This destruction of lamellocytes can account for the reduced frequency of lamellocytes reported for parasitized hosts rather than suppression of lamellocyte differentiation. ${ }^{29}$ 
The factor responsible for destruction of host lamellocytes is contained in the reservoir of an accessory gland of the female wasp reproductive system. ${ }^{27}$ The source of the anti-lamellocytic factor in the female wasp was identified by injected macerated samples of various regions of the female reproductive system into Drosophila larvae and sampling the hemolymph of the injected larvae for the presence of bipolar lamellocytes. Only the contents of the long gland reservoir induced bipolar lamellocytes. ${ }^{27}$ Identification of the active component in reservoir fluid was accomplished by fractionating reservoir fluid on density gradients and treating lamellocytes in vitro with gradient fractions. ${ }^{28}$ Only fractions containing viruslike particles (VLPs) affected lamellocyte morphology. Therefore, in the experiments using reservoir fluid, it should be understood that the VLPs are the active factor in this fluid.

Reservoir fluid can be added to lamellocytes in vitro to follow the modifications in lamellocyte morphology and adhesiveness. ${ }^{44}$ The elongating process of individual lamellocytes in vitro can be observed for 1 to 2 days in axenic medium as the bipolar cells continue to elongate until they become threadlike. Breakage of elongated lamellocyte remnants in undisturbed samples supports the conclusion that lamellocyte destruction in parasitized larvae occurs as the elongating tips of the bipolar cells pinch off. ${ }^{27}$

\section{CYTOSKELETAL MODIFICATIONS IN LAMELLOCYTES}

Cell shape is determined by the disposition of cytoskeletal elements. It was, therefore, expected that lamellocyte transition from round to bipolar involved rearrangement of microtubules. To compare microtubules of lamellocytes in unparasitized and parasitized hosts, indirect immunofluorescence studies with anti-tubulin were used. ${ }^{27}$ The microtubules in round lamellocytes were dispersed in a radial pattern, whereas the microtubules in bipolar lamellocytes were arranged in parallel arrays along the elongating axis of the cells. When lamellocytes were treated simultaneously with wasp reservoir fluid and Vinca alkyloids which depolymerize microtubules to tubulin subunits, the cells retained the discoidal shape rather than assuming a bipolar configuration. ${ }^{45}$ These observations were interpreted as evidence that the elongation process induced by the wasp factor requires depolymerization and repolymerization of tubulin.

A time course ultrastructural study of lamellocytes treated with wasp reservoir fluid in vitro was undertaken to follow events in the reorganization of the cytoskeleton. The methods for the study followed those reported previously. ${ }^{28}$ Briefly, hemolymph from $42 g t w^{l}$ tu-Sz ${ }^{\text {ts }} / y$ sc gt $^{\text {XIIa }}{ }^{81 i x}$ tu-Sz $^{\text {ts }}$ Drosophila larvae were collected in $1.4 \mathrm{ml}$ of phosphate-buffered saline for Drosophila. Reservoir fluid from $35 \mathrm{~L}$. heterotoma females was added to $900 \mu \mathrm{l}$ of the hemocyte sample, and the remainder of the sample was retained as the control sample. A $135-\mu$ l aliquot $\left(C_{0}\right)$ of hemocytes was fixed in $2.5 \%$ glutaraldehyde in cacodylate buffer before reservoir fluid was added and another $135-\mu \mathrm{l}$ aliquot $\left(\mathrm{T}_{0}\right)$ was fixed immediately after the addition of reservoir fluid. Aliquots from the hemocyte mixtures were fixed 10, 30, 60,120 , and 180 minutes later. The cells in the samples were pelleted, postfixed in osmium, and embedded in epon. Thin sections from the epon blocks were stained with uranyl acetate-lead citrate and examined in the electron microscope. 
Ultrastructural characteristics of lamellocytes from larvae with the genotype given above and processed by the same procedures used for the present study have been described previously. ${ }^{28}$ In thin sections the lamellocytes can be recognized by their flat shape and a heavy, electron-dense deposit along the nuclear membrane. Microtubules are concentrated heavily near the nucleus of the lamellocyte. In thin sections the microtubules are dispersed in a helter-skelter fashion (FIG. 1a). Microtubules are prominent in lamellocytes incubated for 10 minutes with reservoir fluid in the medium, but few microtubules are found in the vicinity of the lamellocyte nucleus after treatment with reservoir fluid for 30 minutes (FIG. 1b). Compared with the control lamellocytes, these microtubules are very short in length.

After exposure to reservoir fluid for 60 minutes, long microtubules extend from the vicinity of the nucleus along the elongating axis of the lamellocyte (FIG. 2a). By 180 minutes posttreatment, parallel bundles of microtubules are seen in the lamellocytes (FIG. 2b). The ultrastructural observations thus confirm that wasp reservoir fluid initiates depolymerization and repolymerization of microtubules as the cells change shape from round to bipolar.

The antimitotic drug taxol has been used to study the mechanisms involved in polymerization and depolymerization of microtubules. The drug binds specifically and reversibly to polymerized tubulin and stabilizes microtubules to cold, calcium, and microtubule-disrupting drugs either by lowering the rate of dissociation or by decreasing the rate of steady-state tubulin flux. ${ }^{40-48}$ When cells are incubated in taxol, thr $\mathrm{ie}$ is a redistribution of microtubules that is characterized by the formation of microtubule bundles. ${ }^{49}$ It has also been reported that increased tubulin synthesis in ta sol-treated cells occurs as the result of an increase in tubulin mRNA. ${ }^{49}$

To determine whether taxol interferes with the depolymerization of lamellocyte microtubules induced by wasp reservoir fluid, Drosophila hemocytes in vitro were exposed to $2 \times 10^{-5} \mathrm{M}$ taxol in the presence of wasp material. Two series of experiments were undertaken. In one experiment taxol was mixed with Drosophila $h \in$ mocytes, and wasp reservoir fluid was added to aliquots removed from this sample im mediately $0,15,30$, and 45 minutes later. In a second experiment, Drosophila heinocytes were incubated with wasp reservoir contents, and taxol was added to aliq'xots removed from the hemocyte sample after $0,15,30$, and 45 minutes. Control samples containing only reservoir fluid or taxol were prepared for both experiments. The cell samples were examined by phase microscopy and photographed 4 to 4.5 hours iater.

Lamellocytes incubated in taxol for 4.5 hours retained the typical circular, thin, flat she $\mathrm{se}$ of lamellocytes seen in freshly prepared hemocyte samples and in samples that have been incubated in phosphate-buffered saline or Schneider's culture medium for Drosophila cells (FIG. 3a). The lamellocytes in the samples containing wasp reservoir fluid for 4.5 hours exhibited the elongated, bipolar shape reported in previous in vitro studies (FIG. $3 b$ ).

When reservoir fluid was added immediately after taxol was placed in the hemocyte sample and the cells were examined four hours later, many long, thin projections extended in all directions from the lamellocyte surfaces (FIG. 4a). The typical bipolar transition induced by reservoir fluid was blocked by the presence of taxol in the incubation mixture. In the samples to which reservoir fluid was added at 15 minutes, lamellocytes with multiple surface projections and blebs at the tips were 


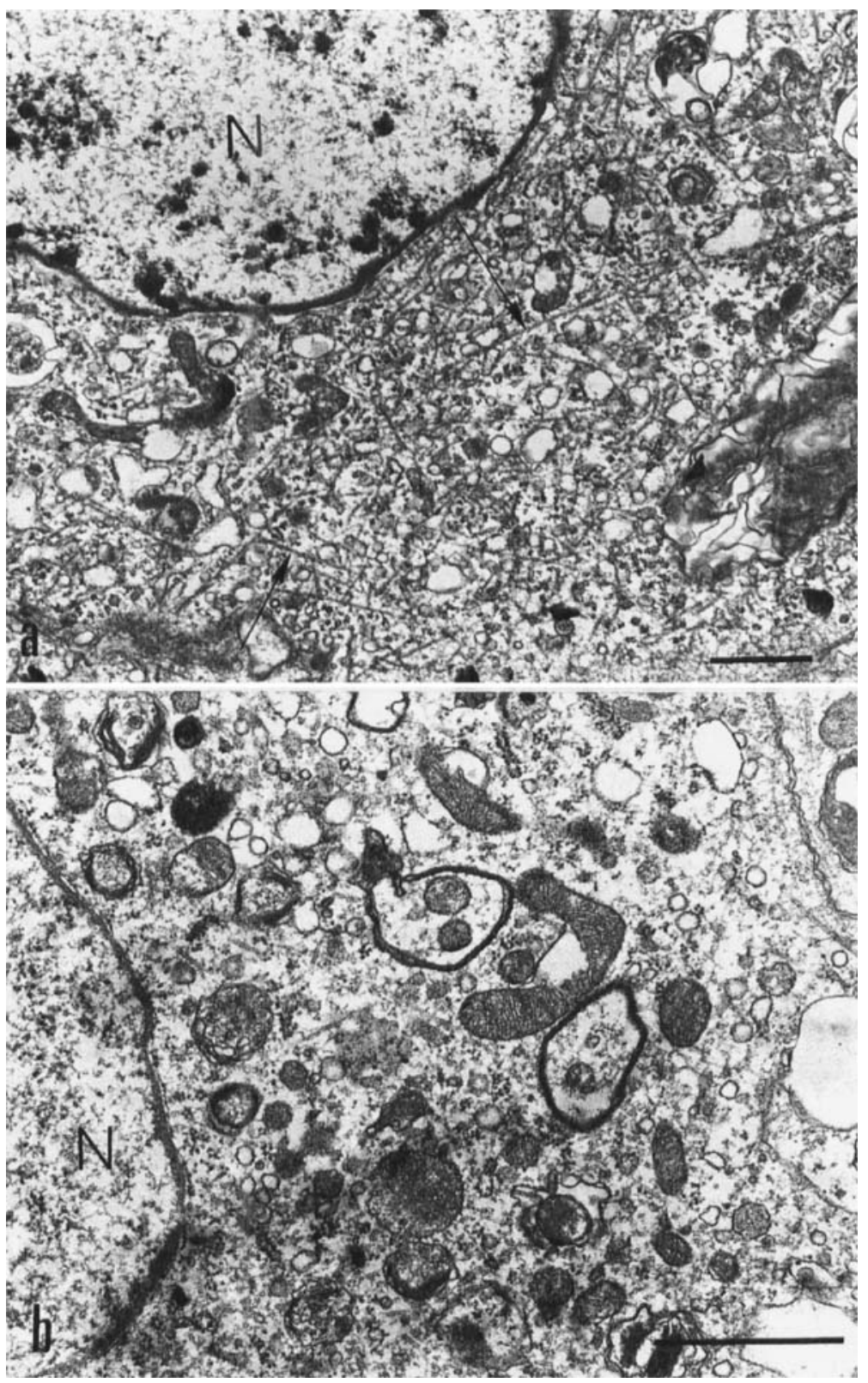

FIGURE 1. Electron micrographs of Drosophila lamellocytes. (a) Many microtubules (arrows) are apparent in the cytoplasm near the nucleus $(\mathrm{N})$ in this lamellocyte from a control sample. Bar $=1 \mu \mathrm{m}$. (b) After 30 minutes' exposure to VLPs, very few short segments of microtubules are apparent in a similar region of a lamellocyte. The cell was photographed at higher magnification than (a) to resolve microtubules. Bar $=1 \mu \mathrm{m}$. 

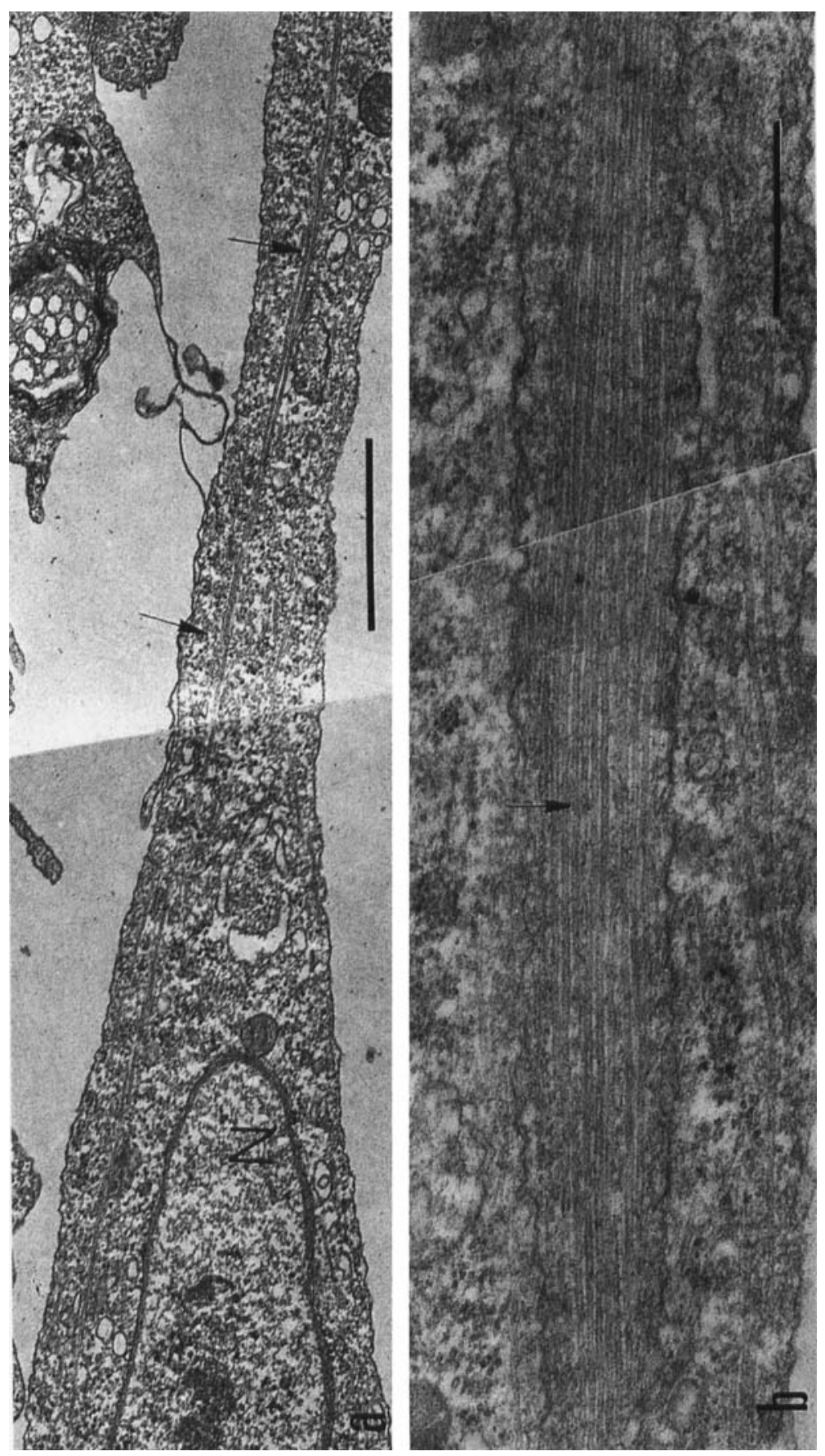

至它

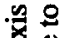

要

号灵

要的

응

声.

.

용

象

8 迅

氖

ลิ8

혼혼

5.500

월 흥

है है

를

肯

范

造

$8 \%$

焉

要青

ธ동를

象点.

os.

5 参

호를

䠉

马

㩆

\%

焉荅

궁

은

悬总

的

伺

을.

马ू “

ㄱ.

되 증

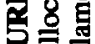

끌 

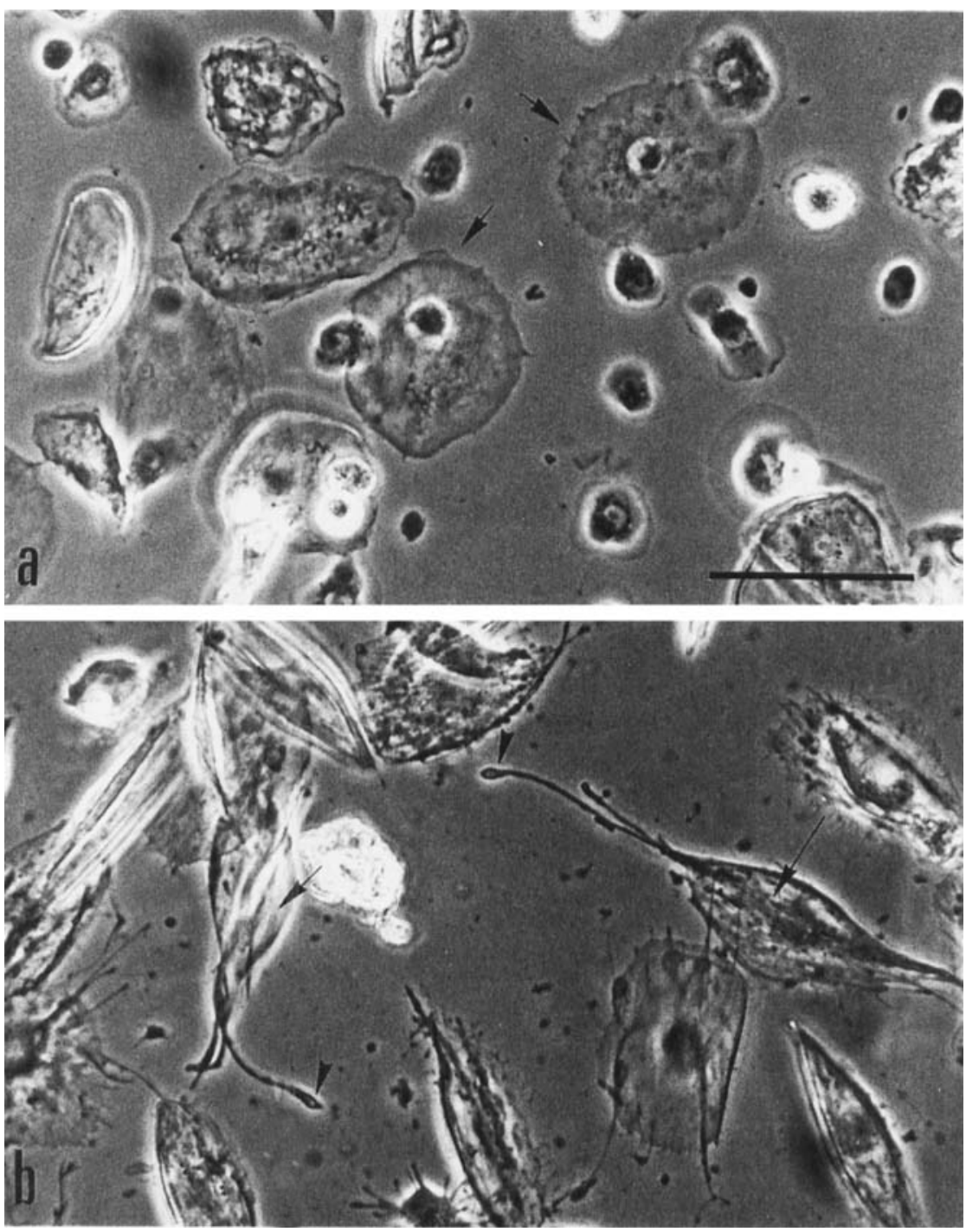

FIGURE 3. (a) Phase micrograph of a hemocyte sample treated with taxol for 4.5 hours. The lamellocytes (arrows) remain discoidal. Bar $=50 \mu \mathrm{m}$. This same magnification is used for all photographs in Figures 3, 4, and 5. (b) Hemocyte sample treated with VLPs for 4.5 hours. Note the elongated bipolar lamellocytes (arrows). There are cytoplasmic blebs (arrowheads) at the elongating tips. In electron micrographs the blebs contain numerous vesicles of various sizes. 
also abundant (Fig. 4b). The surface projections from some of the lamellocytes appeared to be slightly shorter than those in the 0 -minute sample. There was a noticeable decrease in the number of surface projections as well as the length of the projections when reservoir fluid was added 30 minutes after taxol (FIG. $4 c$ ). In the 45-minute sample, projections from the lamellocyte surfaces were very short, and fewer lamellocytes had the surface extensions (FIG. 4d).

In the experiment reversing the order of treatment with reservoir fluid and taxol, the sample in which reservoir fluid and taxol were added initially had lamellocytes with long surface projections, as expected from the results of the first experiment (FIG. 5a). There was a slight reduction in projection length when taxol addition was 15 minutes after reservoir fluid, and an indication of bipolar shape was apparent in some lamellocytes (FIG. 5b). Many bipolar lamellocytes were present in the sample in which taxol treatment followed reservoir fluid by 30 minutes (FIG. 5c). Some of the bipolar lamellocytes had many elongated projections, however. When taxol addition was delayed for 45 minutes, lamellocytes with the typical bipolar effect of wasp reservoir fluid were found (FIG. 5d).

The asteriated lamellocytes produced by treatment with taxol and reservoir fluid must result from microtubule elongation at numerous sites. Because taxol stabilizes microtubules, it must block or reduce the microtubule depolymerization that is initially promoted by VLPs. The elongation of microtubules that is the second function of VLPs must not be affected in the presence of taxol, so elongation occurs at the numerous randomly oriented, taxol-stabilized microtubule ends. The result is a lamellocyte with many surface projections. The length of the projections is a function of time of treatment with taxol. As microtubule stabilization proceeds, the free tubulin pool will be depleted, and tubulin will not be available for microtubule elongation. Therefore, the longer the delay in adding wasp reservoir fluid, the shorter the projections. The depolymerization and reorientation of microtubules induced by the $L$. heterotoma factor occurs within 30 minutes in vitro and is not reversible, because addition of taxol at 30 and 45 minutes did not inhibit the establishment of lamellocyte bipolarity. Our interpretation of the combined treatment of lamellocytes with taxol and reservoir fluid is based on the extensive studies of Horwitz ${ }^{49}$ and her analyses of taxol effects on microtubules.

\section{SPECIFICITY OF VLPS FOR LAMELLOCYTES}

Density gradient-purified VLPs have an outer electron-dense, asymmetric coat with surface projections and a convoluted region that forms a vesicular invagination opposite the thickest portion of the electron-dense material (FIGs. 6a and 8c). When purified VLPs are added to hemolymph samples in vitro, the particles adhere to lamellocyte surfaces. The surface spikes of the VLP are often seen attached to the lamellocyte surface. There appears to be a disruption in the lamellocyte plasma membrane and a slight blistering at the contact site of the VLP projection (FIG. 6b). This suggests that a lytic factor at the tip of the VLP spike may dissolve the cell membrane when contact is made. Sections through VLP spikes often have a clubshaped swelling with an electron-transparent interior (FIG. 6c), which may be the source of lytic factors. 


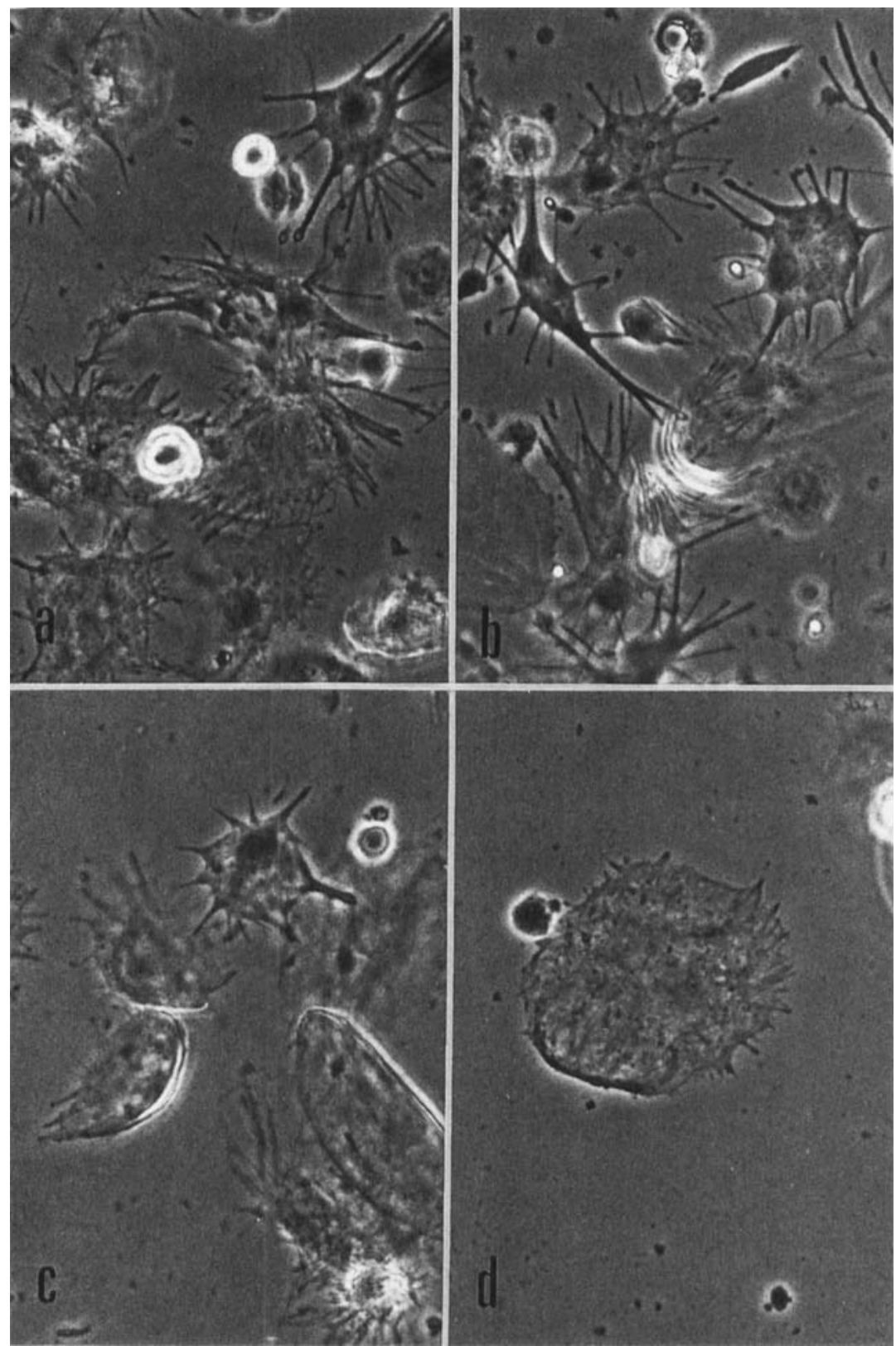

FIGURE 4. Lamellocytes treated with taxol and VLPs. Reservoir fluid was added to the sample immediately after taxol in frame a, after 15 minutes in frame $\mathbf{b}$, after 30 minutes in c, and after 45 minutes in frame $d$. Note the decreasing length of the surface projections as the time interval between taxol treatment and VLP addition increased. 


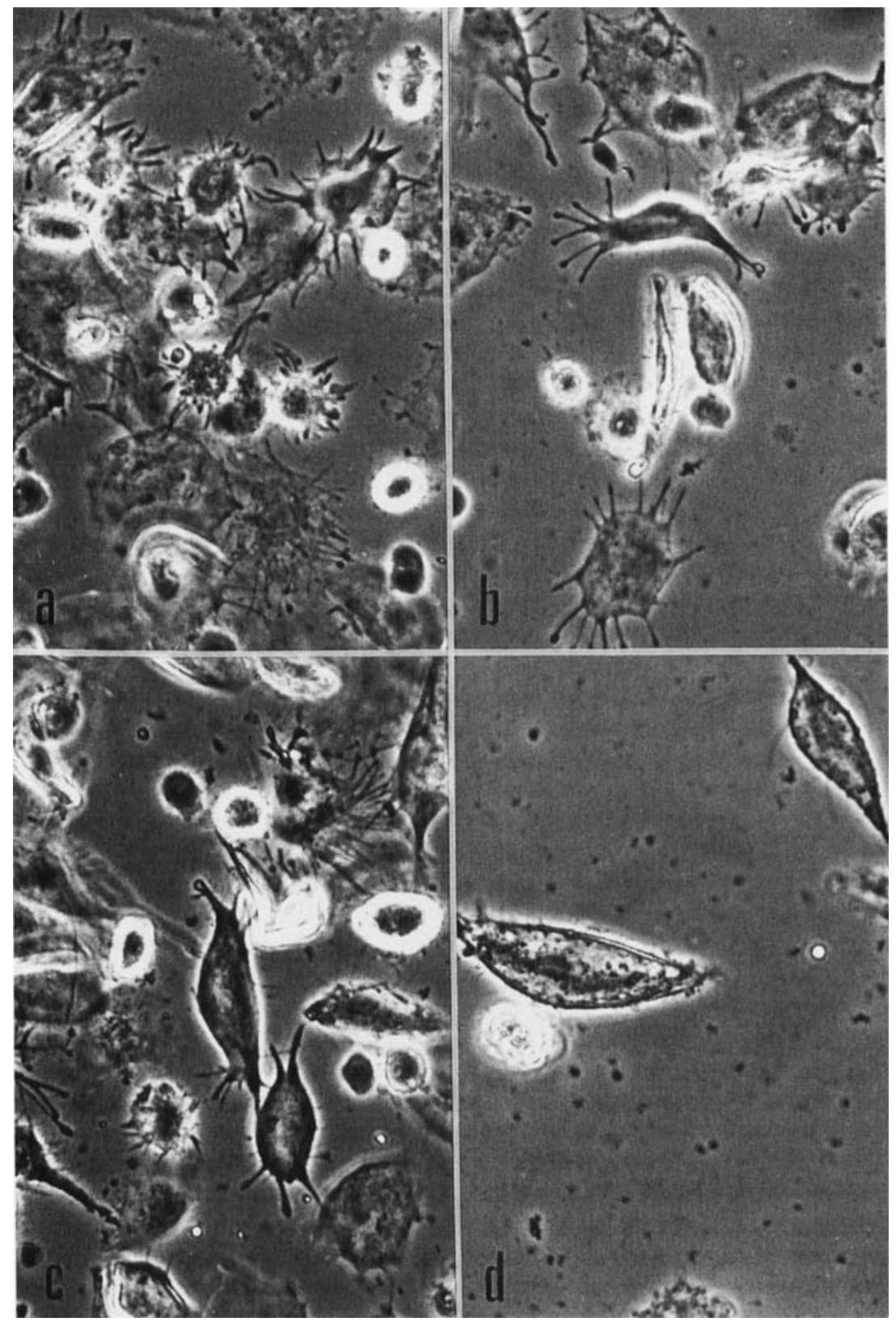

FIGURE 5. Phase micrographs of hemocytes showing the effects of VLPs and taxol. The cells were exposed to reservoir fluid, then taxol was added at $\mathbf{0}$ minutes (frame a), after 15 minutes (frame b), after 30 minutes (frame c), and after 45 minutes (frame d). Blebs can be seen at the tips of the cytoplasmic projections of lamellocytes in frames $\mathbf{b}$ and $\mathbf{c}$. The smaller cells, showing a pronounced phase halo and lacking surface projections, are plasmatocytes. 


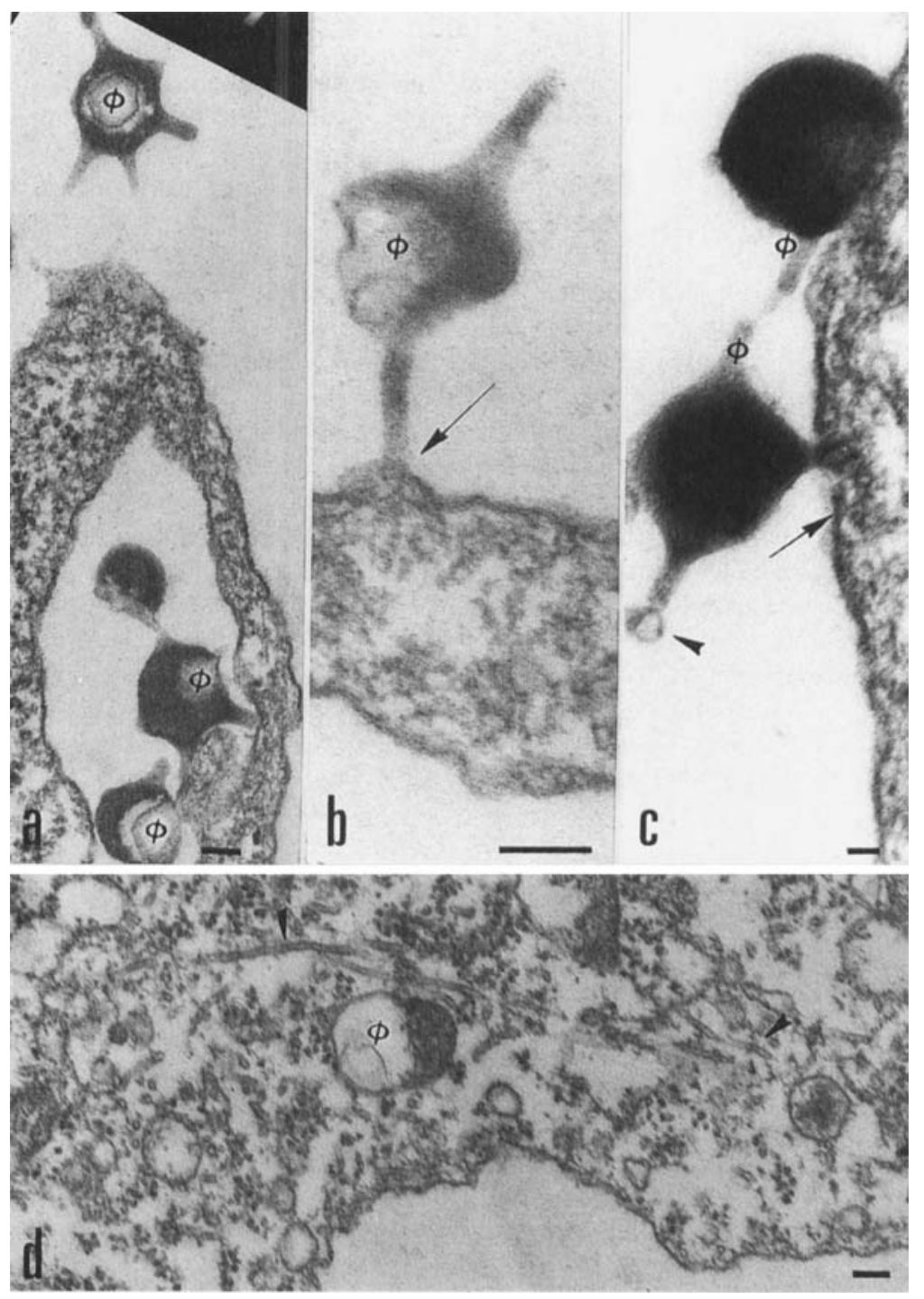

FIGURE 6. Electron micrographs of lamellocyte samples treated with VLPs $(\phi)$. The bar in each frame represents $0.1 \mu \mathrm{m}$. (a) Four VLPs near the surface of a lamellocyte that has folded, presumably during the pelleting or fixation processes. The upper VLP shows five surface spikes, the electron-dense outer region, and the internal electron-transparent cavity. The other VLPs in the frame illustrate the asymmetric organization of the electron-dense material of the particles. (b) Initial contact (arrow) of the VLP spike with the lamellocyte surface resulting in a blister or lysis of the plasma membrane. (c) The spike of the lower VLP in this frame is penetrating the lamellocyte and the continuity of the plasma membrane (arrow) is interrupted. The tip (arrowhead) of the VLP spike has a bleb. The main body of the other VLP is entering the cell. (d) A VLP free in the cytoplasm of a lamellocyte. Microtubules are indicated by arrowheads. 
The entire VLP penetrates the lamellocyte surface without being enveloped by cell surface membranes. The particles are found free in the cytoplasm. No endocytic membrane surrounds the VLPs in the cytoplasm of the lamellocyte (FIG. 6d). Lamellocytes are not phagocytic, ${ }^{50}$ and no evidence for VLP uptake by phagocytosis was seen for lamellocytes.

VLPs in the culture medium were phagocytosed by plasmatocytes and were found in phagocytic vesicles in these hemocytes (FIG. 7a). Destruction of VLPs in phagosomes of plasmatocytes was also seen (FIG. 7b), but no free VLPs were found in the cytoplasm of plasmatocytes. The attachment of the VLP to the lamellocyte surface and its penetration into the cell after dissolution of the plasma membrane suggest that the binding of the VLP surface spike uses a receptor site on the lamellocyte surface. Presumably, this receptor is absent in the plasmatocyte membrane and also in the inner surface of the phagosome membrane, so VLP contact with the plasmatocyte surface initiates the formation of VLP-resistant phagosomes. VLPs are confined to the phagosomes of plasmatocytes where they are inactivated or degraded. No harmful effects of VLPs on plasmatocytes have been observed. In lamellocytes, the VLPs remain free in cytoplasm. Thus, their destructive activity for the lamellocyte requires a free state in cytoplasm so that microtubule components are affected.

The difference in VLP uptake by plasmatocytes and lamellocytes agrees with earlier observations on the attachment and phagocytosis of bacteria by Drosophila hemocytes. ${ }^{50}$ Bacteria injected into the hemocoel of a Drosophila larva adhere to surfaces of both plasmatocytes and lamellocytes, but are phagocytosed by the former
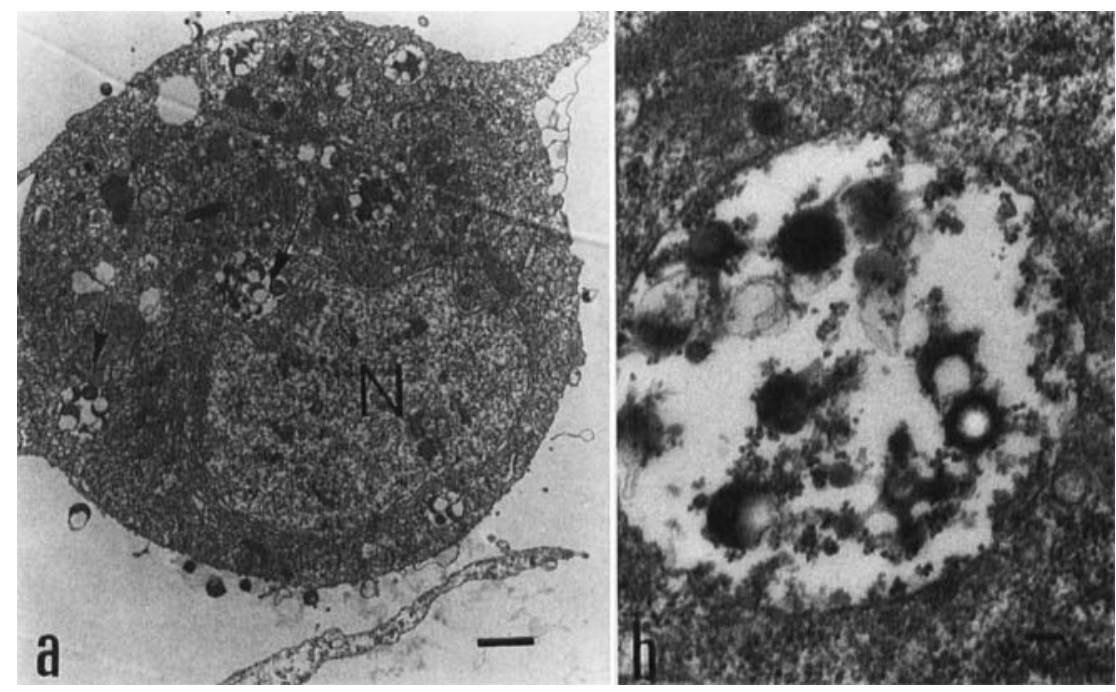

FIGURE 7. (a) A plasmatocyte containing several phagosomes (arrows) with VLPs. Also note the VLPs near the cell surface. $N=$ nucleus. Note the lack of electron-dense material at the nuclear membrane in contrast to the nuclear membrane of the lamellocyte. Bar $=1 \mu \mathrm{m}$. (b) High magnification of a phagosome with VLPs being destroyed. Bar $=0.1 \mu \mathrm{m}$. 
and not the latter. The differentiation of a spherical hemocyte to the discoidal lamellocyte must involve cell surface modifications ${ }^{5 !}$ such that the flattened cells assume adhesive properties required for capsule formation and lose membrane fluidity needed for invagination of phagocytic cups.

The molecular nature of the $L$. heterotoma VLP has not been determined. The mature VLP isolated from the accessory gland reservoir has a complex structure. Some observations have been made on the distribution of particles in the long gland and its storage reservoir in the female wasp. The earliest recognizable stage in the formation of the VLP is found in the proximal region of the gland where large accumulations of 29-nm particles with electron-dense cores are found (FIG 8a). The small particles appear to enlarge and incorporate material that is less electron dense to give a vacuolar appearance to the particles approaching the neck of the gland connected to the duct that opens into the reservoir. Thin sections through the proximal region of the reservoir near its duct opening into the ovipositor contain VLPs with surface projections and an internal element (FIG. 8b) that resemble the gradientpurified VLPs that are active against lamellocytes in vitro (FIG. 8c).

The functions performed by the three types of Drosophila hemocytes are distinct: plasmatocytes are phagocytic, lamellocytes form capsule walls, and crystal cells are required for hardening and blackening reactions of the cellular capsule walls and wound healing. ${ }^{50}$ Of these three cellular defense reactions, encapsulation threatens the survival of a parasite egg floating in the hemocoel. The phagocytic function of plasmatocytes and the melanization reactions of the crystal cells present no imminent danger to the parasite egg. These functions of the plasmatocytes and crystal cells remain intact in parasitized larvae and may even be beneficial in protecting the host against extraneous microbial infections and injury to the body wall. ${ }^{27}$ Nevertheless, it is important that lamellocyte function be disrupted so that the parasite egg remains free of encapsulating hemocytes. This selective and destructive mission is accomplished by the VLPs that the female wasp injects along with its eggs into the host hemocoel.

\section{SUMMARY}

L. heterotoma females inject VLPs along with their eggs into the hemocoel of the Drosophila larva. The VLPs destroy lamellocytes that are potentially harmful for the parasite eggs, but the other types of host hemocytes retain their functions. The parasitized third instar Drosophila larvae continue to grow and pupariate, showing no outward adverse effects. Minimum disruption to the growth and development of the host is advantageous to the endoparasite egg, since survival of the endoparasite depends on the health of the host to the developmental stage at which the endoparasite begins feeding on host tissues.

The target of the VLPs must be microtubule components, because VLP entry into the lamellocyte induces depolymerization and repolymerization of microtubules. Microtubule rearrangement changes the discoidal lamellocyte to a bipolar cell. Concomitant with the modification in cell morphology, lamellocytes lose their surface adhesivity so they cannot adhere to a foreign object or to each other to form a capsule around endoparasite eggs. VLP-affected lamellocytes continue to elongate, 


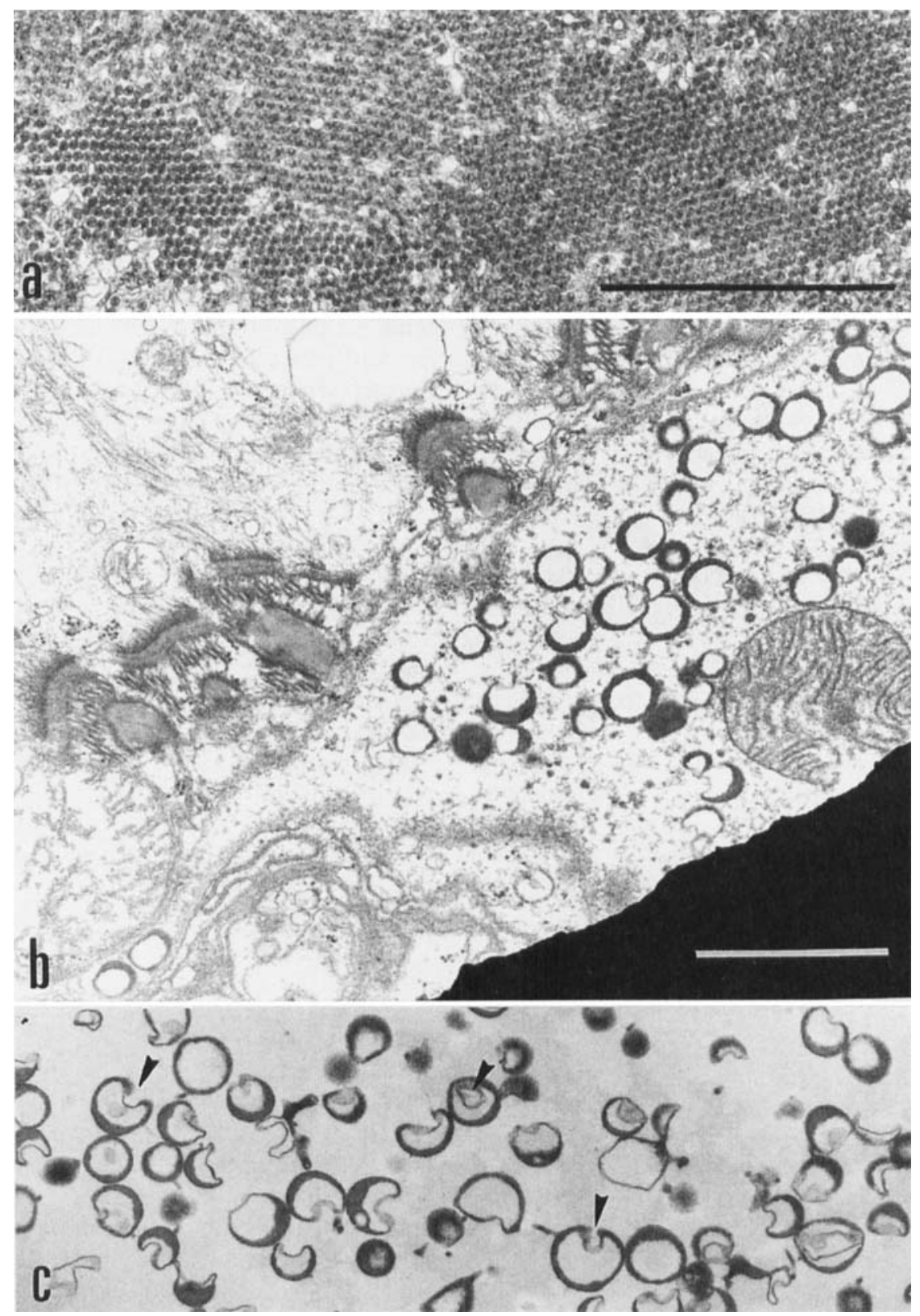

FIGURE 8. (a) Electron micrograph of a section passing through the proximal region of the long gland showing arrays of 29 -nm particles with electron-dense centers. Bar $=1 \mu \mathrm{m}$. (b) $\mathrm{A}$ section passing through the proximal region of the long-gland reservoir near the duct that leads to the ovipositor. Note the profiles of the VLPs in section. Bar $=1 \mu \mathrm{m}$. (c) Section through a pellet of density gradient-purified VLPs. Internal structures can be seen in some of the VLPs, and the invagination can be seen in others (arrowheads). Compare these VLPs with those in frame $b$. Magnification is the same as $b$. 
lose cytoplasmic contents at the poles as blebs full of microvesicles, and are eventually destroyed.

The molecular nature of the L. heterotoma VLP and the mechanisms underlying its specificity for lamellocyte cytoplasm are not known. An interesting consideration is the evolution of a particle whose selectivity for a host hemocyte protects the eggs of the parasitic insect. On the basis of information available at this time, it is clear that the L. heterotoma VLP is a useful tool for studying molecular aspects of microtubules and cytoskeleton.

\section{REFERENCES}

1. SCHELl, S. C. 1952. Trans. Am. Microsc. Soc. 71: 293-302.

2. Poinar, G. O. 1968. Proc. Helminthol. Soc. Washington 35: 161-169.

3. Klomp, H. \& B. J. TeERINk. 1978. Neth. J. Zool. 28: 132-138.

4. Davies, D. H. \& S. B. Vinson. 1986. J. Insect Physiol. 32: 1003-1010.

5. SALT, G. 1973. Proc. R. Soc. London B. 183: 337-350.

6. Bedwin, O. 1979. Proc. R. Soc. London B 205: 267-270.

7. Rotherham, S. 1973. Proc. R. Soc. London B 183: 179-194.

8. Feddersen, I., K. Sander \& O. Schmidt. 1986. Experientia 42: 1278-1281.

9. Berg, R., I. Schuchmann-Feddersen \& O. Schmidt. 1988. J. Insect Physiol. 34: 473480.

10. Salt, G. 1980. Proc. R. Soc. London B 207: 351-353.

11. Wago, H. \& H. Kitano. 1985. Appl. Entomol. Zool. 20: 103-110.

12. Kitano, H. 1986. J. Insect. Physiol. 32: 369-375.

13. Rizki, T. M., R. M. Rizki \& Y. Carton. 1990. Exp. Parasitol. 70: 466-475.

14. Rouault, J. 1979. Compte rendu de l'Académie des Sciences, Paris. 289: 643-646.

15. Carton, Y. \& H. Kitano. 1981. Biol. J. Linn. Soc. 16: 227-241.

16. Osman, S. E. \& E. Führer. 1979. Int. J. Invert. Reprod. 1: 323-332.

17. Tanaka, T. 1982. In The Ultrastructure and Functioning of Insect Cells. H. Akai, R. C. King \& S. Morohoshi, Eds.: 169-172. Society of Insect Cells. Japan.

18. Edson, K. M., S. B. Vinson, D. B. Stoltz \& M. D. Summers. 1981. Science 211: $582-583$.

19. Vinson, S. B., K. M. Edson \& D. B. Stoltz. 1979. J. Invert. Pathol. 34: 133-137.

20. Stoltz, D. B. \& S. B. Vinson. 1979. Can. J. Microbiol. 25: 207-216.

21. Fleming, J. G. W. \& M. D. Summers. 1986. J. Virol. 57: 552-562.

22. Blissard, G. W., J. G. W. Fleming, S. B. Vinson \& M. D. Summers. 1986. J. Insect Physiol. 32: 351-359.

23. Theilmann, D. A. \& M. D. Summers. 1986. J. Gen. Virol. 67: 1961-1969.

24. Stoltz, D. B. \& D. Guzo. 1986. J. Insect Physiol. 32: 377-388.

25. Davies, D. H., M. R. Strand \& S. B. Vinson. 1987. J. Insect Physiol. 33: 143-153.

26. Strand, M. R. \& T. Noda. 1991. J. Insect Physiol. 37: 839-850.

27. Rizkı, R. M. \& T. M. Rizkı. 1984. Proc. Natl. Acad. Sci. USA 81: 6154-6158.

28. Rizki, R. M. \& T. M. Rizki. 1990. Proc. Natl. Acad. Sci. USA 87: 8388-8392.

29. RızKı, T. M. \& R. M. RızKı. 1992. Dev. Comp. Immunol. 16: 103-110.

30. Tanaka, T. 1987. J. Insect Physiol. 33: 413-420.

31. Webi, P. A. \& M. D. Summers. 1990. Proc. Natl. Acad. Sci. USA 87: 4961-4965.

32. Rızкı, T. M. \& R. M. Rızkı. 1984. In Insect Ultrastructure. Vol. 2. R. C. King \& H. Akai, Eds. Plenum Press. New York.

33. RıZKı, T. M. \& R. M. RıZKı. 1986. In Hemocytic and Humoral Immunity in Arthropods. A. P. Gupta, Ed. John Wiley. New York.

34. RIZKı, T. M. 1957. J. Morphol. 100: 437-458.

35. RIZKI, T. M. 1962. Am. Zool. 2: 247-256.

36. RIZKI, T. M. 1957. J. Morphol. 100: 459-472. 
37. Rızkı, R. M. \& T. M. RızKı. 1979. Differentiation 12: 167-178.

38. Rızkı, R. M. \& T. M. Rizkı. 1980. Wilhelm Roux' Arch. 189: 207-213.

39. Rızkı, T. M. \& R. M. Rızkı. 1959. J. Biophys. Biochem. Cytol. 5: 235-240.

40. RizKI, T. M., R. M. Rizki \& E. H. Grell. 1980. Wilhelm Roux' Arch. 188: 91-99.

41. RızKI, R. M. \& T. M. Rızkı. 1990. J. Insect. Physiol. 36: 523-529.

42. WALker, I. 1959. Rev. Suisse Zool. 66: 569-632.

43. NAPPI, A. J. 1975. Nature (London) 255: 402-404.

44. Rizki, R. M. \& T. M. Rızkı. 1991. J. Exp. Zool. 257: 236-244.

45. RızKı, R. M. \& T. M. RızKı. 1990. Experientia 46: 311-315.

46. Parness, J. \& S. B. Horwitz. 1981. J. Cell Biol. 91: 479-487.

47. Schiff, P. B. \& S. B. Horwitz. 1980. Proc. Natl. Acad. Sci. USA 77: 1561-1565.

48. Mole-Bajer, J. \& A. S. Bajer. 1983. J. Cell Biol. 96: 527-540.

49. Horwitz, S. B., L. Liao, L. Greengerger \& L. Lothstein. 1988. In Resistance to Antineoplastic Drugs. D. Kessel, Ed.: 109-125. CRC Press. Boca Raton, FL.

50. RizKI, T. M. \& R. M. RizKI. 1980. Experientia 36: 1223-1226.

51. Rizkı, T. M. \& R. M. Rızkı. 1983. Science 220: 73-75. 